\title{
Nanopatterning of silicon surfaces by low-energy ion-beam sputtering: dependence on the angle of ion incidence
}

\author{
R Gago ${ }^{1,4,5}$, L Vázquez ${ }^{1}, \mathbf{R}$ Cuerno ${ }^{2}$, M Varela $^{3}$, C Ballesteros ${ }^{3}$ \\ and J M Albella ${ }^{1}$ \\ ${ }^{1}$ Instituto de Ciencia de Materiales de Madrid (CSIC), 28049 Cantoblanco, Madrid, Spain \\ 2 Departamento de Matemáticas and GISC, Universidad Carlos III de Madrid, \\ Avenida Universidad 30, 28911 Leganés, Madrid, Spain \\ ${ }^{3}$ Departamento de Física, Universidad Carlos III de Madrid, Avenida Universidad 30, \\ 28911 Leganés, Madrid, Spain \\ E-mail: R.Gago@fz-rossendorf.de
}

\begin{abstract}
We report on the production of nanoscale patterning on Si substrates by low-energy ion-beam sputtering. The surface morphology and structure of the irradiated surface were studied by atomic force microscopy (AFM) and high-resolution transmission electron microscopy (HRTEM). Under ion irradiation at off-normal incidence angle $\left(\sim 50^{\circ}\right)$, AFM images show the formation of both nanoripple and sawtooth-like structures for sputtering times longer than $20 \mathrm{~min}$. The latter feature coarsens appreciably after 60 min of sputtering, inducing a large increase in the surface roughness. This behaviour is attributed to the preferential direction determined on the substrate by the ion beam for this incidence angle, leading to shadowing effects among surface features in the sputtering process. Under irradiation at normal incidence, the formation of an hexagonal array of nanodots is induced for irradiation times longer than $2 \mathrm{~min}$. The shape and crystallinity of the nanodots were determined by HRTEM. At this incidence angle, the surface roughness is very low and remains largely unchanged even after $16 \mathrm{~h}$ of sputtering. For the two angle conditions studied, the formation of the corresponding surface structures can be understood as the interplay between an instability due to the sputtering yield dependence on the local surface curvature and surface smoothing processes such as surface diffusion.
\end{abstract}

\section{Introduction}

The production of nanocrystalline semiconductor structures has awakened great interest due to their application in optoelectronic and electronic devices. In particular, for siliconbased devices, this interest is enhanced by the lack of an optical emitter compatible with standard Si-based ultra-large-scaleintegration (ULSI) technology [1]. Silicon has an indirect energy bandgap and, therefore, is highly inefficien as a light source. However, light-emitting properties from $\mathrm{Si}$ are

\footnotetext{
4 Author to whom any correspondence should be addressed.

5 Present address: Forschungszentrum Rossendorf eV, Postfach 510119, 01314 Dresden, Germany.
}

obtained in quantized systems [2], where the typical lengths are around a few nanometres. Thus, findin a procedure for the controlled production of semiconductor, particularly silicon, nanostructures remains a key requirement for the development of future optoelectronic and electronic devices. Among the different techniques currently employed for the production of semiconductor nanostructures, ion-beam sputtering (IBS) has recently proved to be an attractive option [3-12].

Different nanostructures can be produced on a semiconductor surface by IBS depending on the incidence angle. Specificall, both under normal incidence [3] and under offnormal incidence but with simultaneous substrate rotation [4], IBS induces the formation of hexagonal arrays of nanodots. 
The formation of nanodots by IBS has been reported on $\mathrm{GaSb}$ [3], InP [4] and, recently, on Si by the present authors [5]. The production of ordered arrays of nanodots on $\mathrm{Si}$ and $\mathrm{Ge}$ is important, since it can open the way to fabricating nanostructures on the technologically relevant group IV semiconductor materials. Such structures could be applied in the production of quantum dots for quantum devices as well as of black surfaces for optoelectronic and photovoltaic applications [3]. The possibility in which nanodot formation might be achieved on epitaxial silicon layers grown on a higherbandgap substrate would be of particular relevance. In such a system quantum confinemen effects might be observed in the photoluminiscence emission spectra. It is worth mentioning that such a property has already been reported for $\mathrm{GaSb} / \mathrm{AlSb}$ bombarded under normal-incidence conditions [3]. In contrast, for off-normal incidence a nanoripple morphology is induced [6], the orientation of the ripple grooves being parallel or perpendicular to the ion-beam direction for incidence angles to the substrate normal below and above a critical value (usually in the $60^{\circ}-70^{\circ}$ range), respectively [7]. Experimentally, ripple patterning has been observed in amorphous materials [8], metals [9] and semiconductors [10-12]. Another interesting technological application of both nanodot and nanoripple-induced morphologies is the possibility of using them as templates for the serial production of controlled nanopatterns on polymeric materials [13].

The production of these nanostructures by IBS is based [14-16] on the interplay between the sputtering processes, which depend on the local surface curvature, and surface diffusion smoothing processes, which depend on the surface temperature. For the technological applications mentioned, control of size, uniformity, roughness and spatial ordering of the different morphologies is required. Therefore, a systematic study of the dependence of these properties on process parameters is required in order to understand completely the underlying physical mechanisms and to gain complete control of the technique. Thus, in this paper, we address the study of the temporal evolution of ripple and dot morphologies on $\mathrm{Si}$ surfaces (i.e. their dependence on the ion dose) with the aim of characterizing the features of the different nanopatterns appearing, as well as their dependence on the ion dose and the angle of incidence. This kind of quantitative and qualitative analysis is needed in order to be able to fabricate in the future, in a controlled way, a given nanopattern on a silicon surface. In this sense, the study of the different morphological behaviours, namely their corresponding temporal and length scales, becomes crucial for any attempt to fabricate nanopattern structures, since it allows us to identify the physical mechanisms responsible for them [14-16], which determine the dynamics of the system.

\section{Experiment}

The samples were processed in a high-vacuum chamber with a base pressure of $2 \times 10^{-7}$ mbar. In all our studies, we used single-crystal n-type $\mathrm{Si}(100)$ substrates (phosphorus doped and $0.3-1 \Omega \mathrm{cm}$ ). Prior to entering the chamber, the substrates were cleaned sequentially in ultrasonic baths with trichloroethylene, acetone and methanol. The ion beam was provided by a commercial $3 \mathrm{~cm}$ Kauffman ion gun. The samples were irradiated with $1.2 \mathrm{keV} \mathrm{Ar}^{+}$ions at an incidence angle of $50^{\circ}$ with respect to the substrate normal for the production of ripple structures and at normal incidence for nanodot formation. As the etch rate for normal incidence is found to be twice that for $50^{\circ}$ incidence, and remembering that the ion current density is reduced to one-quarter of its normal value, the effective sputtering coefficien at $50^{\circ}$ must be twice that for normal incidence. This result is actually in agreement with the known $\sim \cos ^{-n} \theta(1<n<2)$ dependence of the sputtering yield on the ion incidence angle for non-glancing beams [14, 17]. The experiments were performed at room temperature and the sputtering time, i.e. ion dose, was varied from 1 up to $960 \mathrm{~min}$.

The resulting surface morphology was characterized by atomic force microscopy (AFM) and high-resolution transmission electron microscopy (HRTEM). The AFM measurements (Nanoscope IIIa) were performed in air and in tapping mode, using Si cantilevers with a radius of curvature of $\sim 10 \mathrm{~nm}$. Specimens suitable for HRTEM inspection were prepared by standard procedures and examined using a Philips CM200 FEG analytical microscope operating at $200 \mathrm{kV}$.

\section{Results and discussion}

Figures 1(a) and (b) show the surface morphology and lateral surface profil obtained from the AFM images of two samples bombarded at $50^{\circ}$ off-normal incidence for $20 \mathrm{~min}$ (a) and $180 \mathrm{~min}$ (b). Two morphologies are clearly observed, a smaller rippled one with a wavelength in the range of 30 $50 \mathrm{~nm}$ and amplitude of 2-5 nm and a larger sawtooth-like one with a wavelength in the $200-700 \mathrm{~nm}$ range and amplitude of $50-100 \mathrm{~nm}$. Both structures are oriented perpendicular to the ion beam projection on the substrate surface, which is consistent with theoretical predictions [14]. The smallerwavelength ripple feature dominates the surface morphology for sputtering times shorter than $20 \mathrm{~min}$, while it appears superimposed onto the larger-wavelength sawtooth-like structure for sputtering times longer than $20 \mathrm{~min}$. Regarding the temporal evolution of both features, the ripple structure does not undergo large changes, whereas the amplitude and wavelength of the sawtooth-like structure coarsen with time. In fact, the latter structure determines the relatively high surface roughness $(\sigma)$ observed, which is displayed in figur 2. The morphological evolution of this structure shows a faceting process for $t \geqslant 60 \mathrm{~min}$. This feature can be observed in the profil displayed in the bottom part of figur 1(b), which presents sharp and slanted facets parallel to the ion-beam direction. Given that we have obtained the same angle of the saw-tooth morphology for different substrate orientations with respect to the ion incidence direction, we can conclude that faceting is not related to a specifi crystal orientation but rather to geometrical shadowing. This effect is induced by the growth of the ripple amplitude, and implies that the top part of the ripples shadows the bottom part of the structures from the ion beam, with the effect of relatively larger erosion at surface protuberances and production of slanted faces [18].

It is interesting to note, as derived from the AFM images, that although the sawtooth-like morphology is predominant as the sputtering process proceeds, the nanoripple structure is still present even after $6 \mathrm{~h}$ of ion sputtering. Thus, our data indicate that the silicon substrate should be sputtered for times shorter than $60 \mathrm{~min}$ in order to avoid the formation 

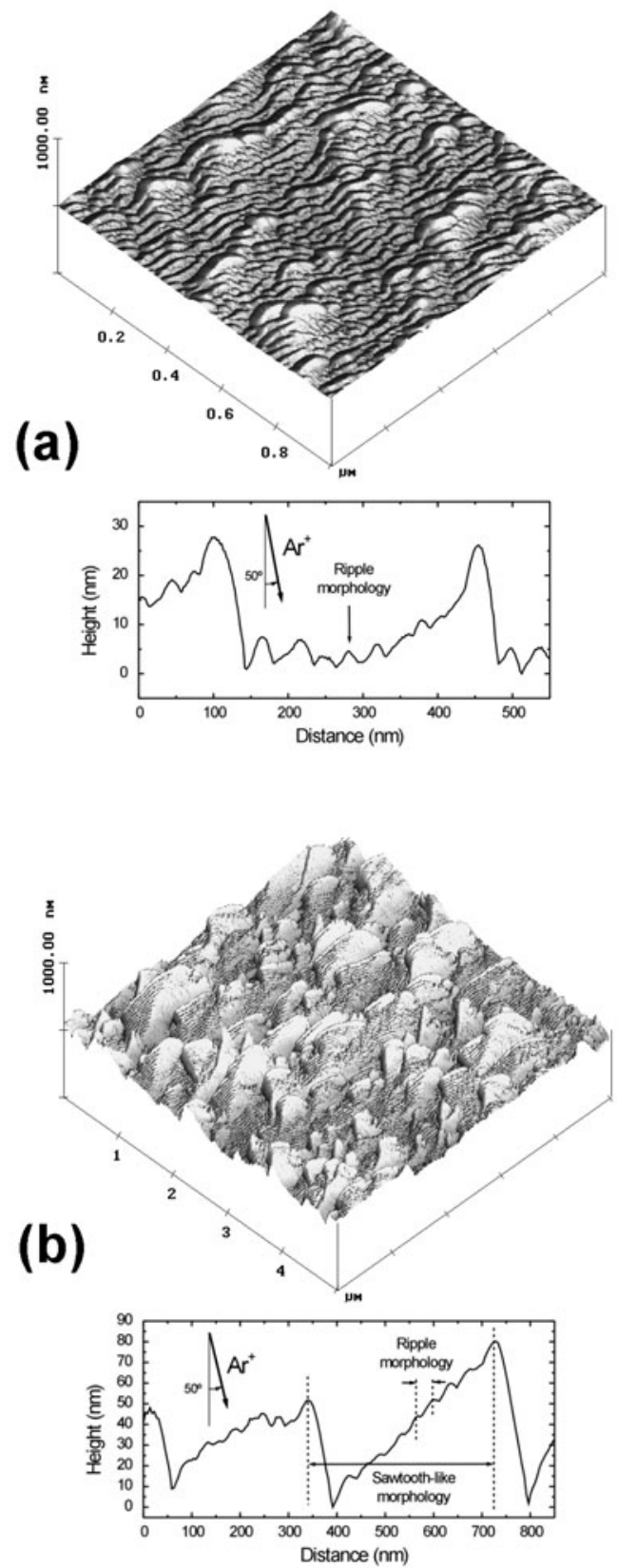
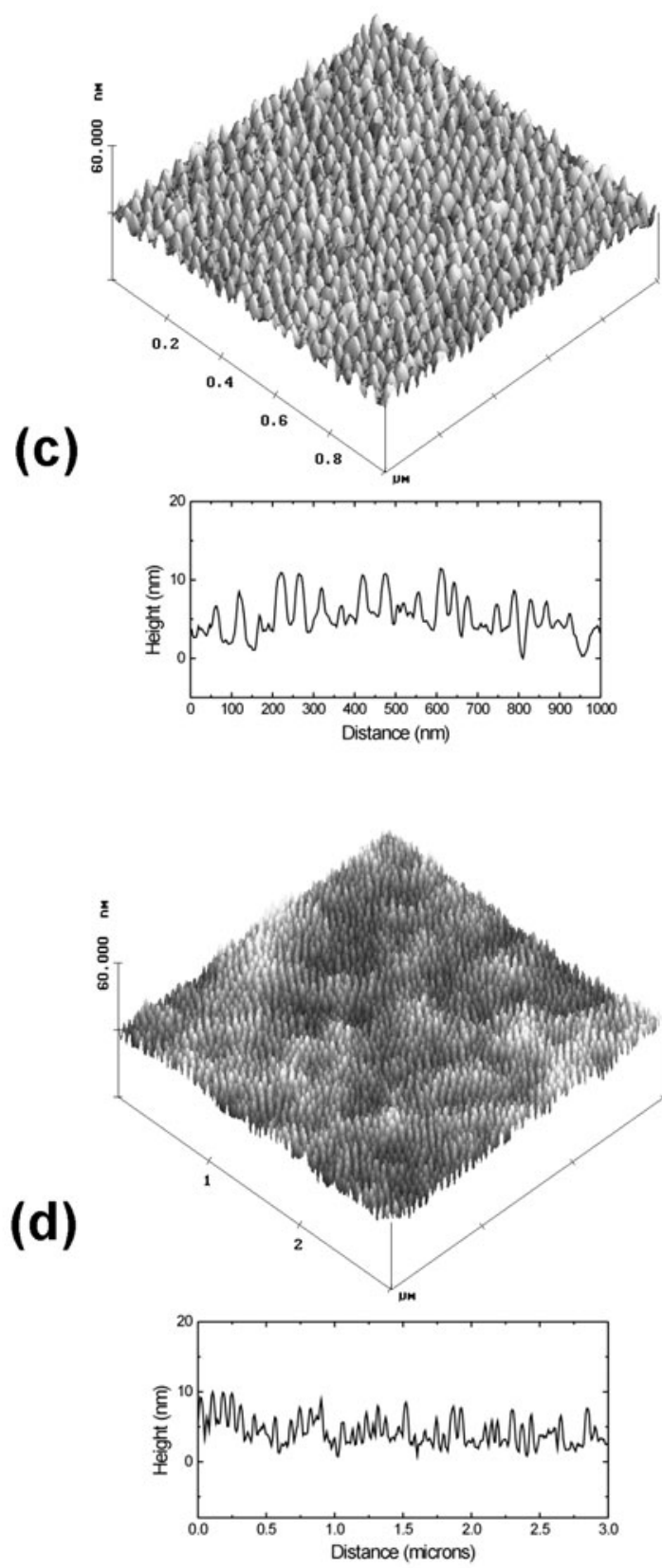

Figure 1. Three-dimensional view of AFM images under (a) off-normal incidence after 20 min, (b) off-normal incidence after 180 min, (c) normal incidence after $10 \mathrm{~min}$ and (d) normal incidence after $960 \mathrm{~min}$. A characteristic surface profil of each AFM image is shown below the corresponding image. In (a) and (b) the surface profil is taken along the projection of the ion-beam direction onto the substrate surface. Note the different scales in the horizontal and vertical axes in the surface profiles

of the rough sawtooth structure and keep the dimensions of the ripple in the nanometre range, which could be important for nanotechnological applications. A complete study of the mechanisms and evolution of the sawtooth-like structure is in progress and will be presented in a future publication [19].

For normal ion incidence, figure 1(c) and (d) show the formation of nanodot structures with a height of $\sim 6 \mathrm{~nm}$ and a diameter of 40-50 $\mathrm{nm}$. The nearest-neighbour distance between dots tends to be a time-independent constant, at least up to $16 \mathrm{~h}$ of ion bombardment. Because of tip convolution effects, the dot shape has to be assessed by HRTEM rather than by AFM. The HRTEM images show that the nanodots are crystalline, except for an amorphous layer $\sim 2 \mathrm{~nm}$ thick on the surface, and with a lenticular shape $(6-7.5 \mathrm{~nm}$ height and $40-60 \mathrm{~nm}$ width) [5]. It is known [20] that residues of low-sputtering material present on the sputtered surface can cause the development of conical features. However, we believe that this effect is not the origin of the nanodot morphology because, first the substrates are subject to a cleaning procedure prior to being mounted into the sputtering chamber. Second, the reproducible self-organization of the pattern seems inconsistent with this effect. Third, recent experiments performed at substrate temperatures of $200^{\circ} \mathrm{C}$, at which the residues present on the substrate surface are more efficientl removed, lead essentially to the same kind of pattern [21]. 


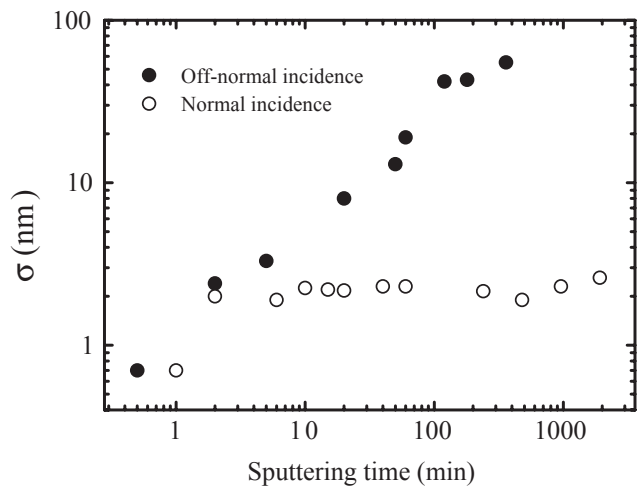

Figure 2. Surface roughness $(\sigma)$ for off-normal $(\bullet)$ and normal $(O)$ ion incidence as a function of the sputtering time.

In addition to this analysis, AFM imaging allows us to study both the self-organization of these nanostructures and their roughness and height distributions. The autocovariance of the AFM images confirm that these nanostructures selforganize into a short-range hexagonal symmetry [5]. Above 20 min of sputtering, a long-wavelength corrugation develops. This feature coarsens with time, being evident in the AFM image sketched in the top part of figur 1(d). From the surface profil shown from this image (bottom part of figur 1(d)), we can see that after $16 \mathrm{~h}$ of sputtering this corrugation is $\sim 500 \mathrm{~nm}$ wide and $<5 \mathrm{~nm}$ high. Despite the coarsening effect, the low amplitude of the corrugation implies that the surface roughness, $\sigma$ (see figur 2 ), is mainly determined by the nanodot structures, since it attains a saturation value once the nanodot structure is formed, i.e. after $\sim 2 \mathrm{~min}$ of sputtering. This result is in clear contrast with the behaviour observed for off-normal sputtering experiments, in which the appearance and further development of the larger structures lead to an evident increase in the roughness. It should be recalled that the etching rate is higher for the normal-incidence configuratio than for the off-normal-incidence set-up. In fact, it is worth mentioning the extremely low value of the surface roughness, $\sim 2 \mathrm{~nm}$, obtained under normal-incidence conditions even after $16 \mathrm{~h}$ of sputtering, i.e. after a layer $\sim 50 \mu \mathrm{m}$ thick of the silicon substrate has been etched away.

Finally, AFM data allow us to study the evolution of the average nanodot height and density with sputtering time. The results are shown in figur 3 . We observe that the nanodot density (a) attains a constant value of $\sim 3.5 \times 10^{10} \mathrm{dots}_{\mathrm{cm}}^{-2}$ after $10 \mathrm{~min}$ of ion sputtering. This trend in the dot density seems to imply a slight coarsening of the nanodot diameter as observed by AFM, although tip convolution effects do not allow us to quantify this precisely. However, the nanodot height average values (b) can be reliably obtained from the AFM data. It is observed that this value lies in the 6-7 nm range for all the samples studied. Considering slight changes, we can say that the height of the dot increases with time until it reaches a maximum value of $\sim 7 \mathrm{~nm}$ around $2 \mathrm{~h}$ of IBS. For times longer than $2 \mathrm{~h}$, the height starts to decrease and then saturates for longer times $(\geqslant 8 \mathrm{~h})$ at $\sim 6 \mathrm{~nm}$. At this point we should consider the role of the substrate heating induced by the impinging ions. This process, which may be slow, could be related to the change in dot density and height, particularly over the firs $10 \mathrm{~min}$ of sputtering. In order to assess the importance of this effect,

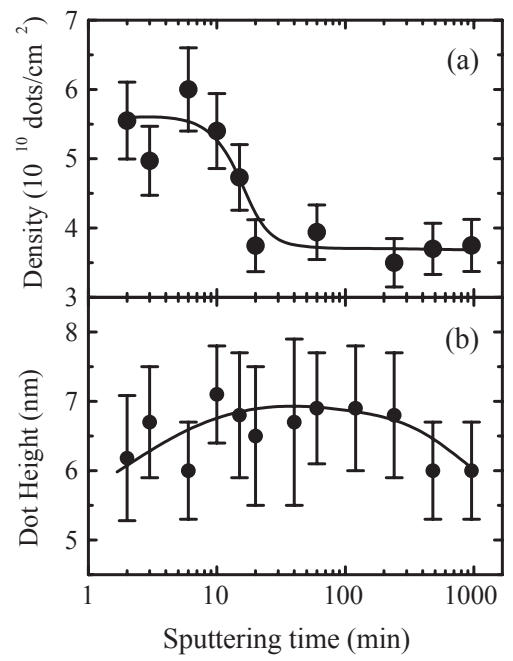

Figure 3. Average dot density (a) and height (b) as functions of the sputtering time for normal incidence. Solid curves are guides to the eye.

experiments on the influenc of the substrate temperature on the nanodot pattern (particularly on the nanodot dimensions) are needed. We expect to report on these experiments shortly.

Summarizing the above observations, we have found rather different behaviours of the surface morphology and dynamics simply when considering two different ion-incidenceangle conditions for a given IBS system. We can compare our observations with the available theories of surface morphology under IBS. It is well known $[14,17]$ that the local erosion depends on the incidence angle, through its anisotropic dependence on the local surface curvature. The anisotropy arises from the physically different roles of the direction on the substrate parallel to the projection of the ion beam and the perpendicular direction. The continuum model of surface dynamics under IBS for amorphous or amorphizable materials by Bradley and Harper (BH) [14] and its generalizations $[15,16]$ account satisfactorily for our observations in the sense that they predict the production of an assembly of dots or pits at normal incidence and a periodic ripple morphology at oblique incidence angles, both of which occur as the result of the interplay between an instability due to the sputtering yield dependence on the local surface curvature and surface diffusion smoothing processes. However, at a more detailed level, there are features of our present experiments which are difficul to explain within the $\mathrm{BH}$ model [14]. We believe that this is due to the failure of the approximations implicit in such a linear model. On the one hand, for normal incidence we observe the nanodot structure to stabilize into a stationary state, while the prediction of the BH model is the occurrence of unbounded amplitude growth. This effect can possibly be accounted for by the nonlinear dependences of the local etching velocity on the surface slopes $[15,16]$. On the other hand, both the BH model [14] and its non-linear extensions [15] assume small surface slopes across the substrate, thus neglecting shadowing effects among different surface features. However, these assumptions are clearly seen to fail in our oblique-incidence experiments. In conclusion, our experiments coincide in a wide sense with the current theoretical understanding of surface dynamics under IBS. However, we believe that our results also point out some of the limitations of the available theoretical models. Further 
developments on this side are clearly desirable in order to completely understand our experimental system, and to understand morphology dynamics under IBS from a general point of view.

\section{Conclusions}

IBS is a suitable technique for the nanopatterning of silicon surfaces. Under off-normal ion incidence, both ripple and sawtooth-like morphologies are produced. The latter coarsens with time, leading to an appreciable increase of the surface roughness. Under normal-incidence conditions, the sputtering process leads to the formation of hexagonal arrays of crystalline nanodots. After 10 min of sputtering the average height of the nanodots, the surface roughness and the surface nanodot density have reached an essentially constant value. The marked differences observed for the time evolution of the two kinds of nanopattern are due to the anisotropy in the ion-beam config uration in the off-normal situation. Comparison of the main qualitative features of our results with the existing theoretical descriptions of IBS allows us to attribute the main morphological features observed to an interplay between an instability intrinsic to the sputtering process and surface diffusion.

\section{References}

[1] Ng W L, Lourenço M A, Gwilliam R M, Ledain S, Shao G and Homewood K P 2001 Nature 410192

[2] Shimizu-Iwayama T, Nakao S and Saitoh K 1994 Appl. Phys. Lett. 651814
[3] Fackso S, Dekorsy T, Koerdt C, Trappe C, Kurz H, Vogt A and Hartnagel H L 1999 Science 2851551

[4] Frost F, Schindler A and Bigl F 2000 Phys. Rev. Lett. 854116

[5] Gago R, Vázquez L, Cuerno R, Varela M, Ballesteros C and Albella J M 2001 Appl. Phys. Lett. 783316

[6] Navez M, Sella C and Chaperot D 1962 C. R. J. Phys. 254 240

[7] Habenicht S, Bolse W and Lieb K P 1999 Phys. Rev. B 60 R2200

[8] Mayer T M, Chason E and Howard A J 1994 J. Appl. Phys. 76 1633

[9] Rusponi S, Boragno C and Valbusa U 1997 Phys. Rev. Lett. 78 2795

[10] Chason E, Mayer T M, Kellerman B K, McIlroy D T and Howard A J 1994 Phys. Rev. Lett. 723040

[11] Carter G and Vishnyakov V 1996 Phys. Rev. B 5417647

[12] Jiang Z X and Alkenade P F A 1998 Appl. Phys. Lett. 73315

[13] Azzaroni O, Schilardi P, Gago R, Vázquez L and Salvarezza $\mathrm{R}$, in preparation

[14] Bradley R M and Harper J M E 1988 J. Vac. Sci. Technol. A 6 2390

[15] Cuerno R and Barabási A-L 1995 Phys. Rev. Lett. 744746

[16] Kahng B, Jeong H and Barabási A-L 2001 Appl. Phys. Lett. 78 805

[17] Cuomo J J, Rossnagel S M and Kauffman H R 1989 Handbook of Ion Beam Processing Technology (Park Ridge, NJ: Noyes) pp 81-2

[18] Carter G 1999 J. Appl. Phys. 85455

[19] Gago R, Vázquez L, Cuerno R and Albella J M, in preparation

[20] Auciello O 1986 Erosion and Growth of Solids Stimulated by Atom and Ion Beams ed G Kiriakidis, G Carter and J L Whitton (Hingham, MA: Nijhoff) pp 394-422

[21] Gago R, Vázquez L, Cuerno R, Varela M, Ballesteros C and Albella J M, in preparation 